\title{
RELIABILITY OF COMPUTER DESIGNED SURGICAL GUIDES IN SIX IMPLANT REHABILITATIONS WITH TWO YEARS FOLLOW-UP
}

\author{
M. Giordano ${ }^{1}$, P. Ausiello ${ }^{2}$, M. Martorelli ${ }^{3}$, R. Sorrentino ${ }^{2}$, D. C. Watts ${ }^{4,5}$ \\ ${ }^{1}$ Giordano Dental Office, Caserta, Italy. \\ ${ }^{2}$ Department of Restorative Dentistry, School of Dentistry, University of Naples Federico II, \\ Naples, Italy. \\ ${ }^{3}$ Department of Mechanics and Energetics, School of Engineering, University of Naples Fede- \\ rico II, Naples, Italy (corresponding - massimo.martorelli@unina.it) \\ ${ }^{4}$ School of Dentistry and Photon Science Institute, University of Manchester, UK \\ ${ }^{5}$ Institute of Materials Science and Technology, Friedrich-Schiller-University Jena, Germany
}

\begin{abstract}
Objective: To evaluate the reliability and accuracy of computer-designed surgical guides in osseointegrated oral implant rehabilitation.

Materials and methods: Six implant rehabilitations, with a total of 17 implants, were completed with computer-designed surgical guides, performed with the master model developed by muco-compressive and muco-static impressions. In the first case, the surgical guide had exclusively mucosal support, in the second case exclusively dental support. For all six cases computer-aided surgical planning was performed by virtual analyses with $3 D$ models obtained by dental scan DICOM data.

The accuracy and stability of implant osseointegration over two years post surgery was then evaluated with clinical and radiographic examinations. Radiographic examination, performed with digital acquisitions (RVG - Radio Video graph) and parallel techniques, allowed two-dimensional feedback with a margin of linear error of 10 percent.

Results: Implant osseointegration was recorded for all the examined rehabilitations. During the clinical and radiographic post-surgical assessments, over the following two years, the peri-implant bone level was found to be stable and without appearance of any complications. The margin of error recorded between pre-operative positions assigned by virtual analysis and the post-surgical digital radiographic observations was as low as $0.2 \mathrm{~mm}$.

Conclusions: Computer-guided implant surgery can be very effective in oral rehabilitations, providing an opportunity for the surgeon: (a) to avoid the necessity of muco-periosteal detachments and then (b) to perform minimally invasive interventions, whenever appropriate, with a flapless approach.
\end{abstract}

Keywords: Surgical guides, Virtual Modeling, Osseointegration, Minimally invasive approach, Muco-compressive impression, Muco-static impression. 


\section{INTRODUCTION}

Oral rehabilitation supported by osseointegrated implants now represents a valuable therapeutic strategy both from an aesthetic and a functional point of view [1-3]. In oral rehabilitation with a traditional surgical approach using osseointegrated implants, the surgeon is often obliged to perform essential muco-periosteal detachments in order to obtain good visibility of the bone structures. Indeed the later intraoperative stages cannot be properly viewed if a flapless approach is used, which, although valid from a point of view of implant osseointegration [4-6], significantly increases the margins of imprecision and the possibility of failure if not performed with a guided technique.

Muco-periosteal detachments turn out to be much more extended when the number of implants to be placed increases and this may result in significant implant mobility for the patient. Furthermore, if strategic positioning of implant inclined fixtures, mesially or distally, is required, compared to anatomicaly relevant structures, the operator is forced to invade the maxillary sinus in order to highlight the margins - or, alternatively, to highlight the emerging part of the inferior alveolar nerve from the mental foramen in the case of a procedure on the mandible.

Intra-operative visibility is important especially if bone structures have been preoperatively evaluated only with the aid of two-dimensional radiographic investigations, such as standard radiographs or intraoral orthopantomographies. These last two procedures, however, expose the patient to a low radiation dosage [7-10]. More information about the quality, quantity and bone topography can be obtained preoperatively with the aid of survey systems such as three-dimensional CT (Computed Tomography) and Cone Beam CT (CBCT) systems [11$14]$.

The data coming from these examinations, however, must be analyzed with the aid of dedicated software. This allows a very detailed interpretation and therefore significantly increases the pre-operative information available to the implant surgeon. In particular, if threedimensional radiographic investigations are conducted with radiological intraoral guides, developed by previous diagnostic waxing, it is then possible to place the implants by virtual simulations with computer-aided planning.

The aims of this work were to reduce the time required for surgery, to increase the accuracy of the implant positioning using a flapless approach, or in any case one that is minimally invasive, and at the same time to improve the post-operative treatment of the patient through the use of computer-designed surgical guides, developed with a combination of virtual models and mechanical systems. By using these it is possible to input and transfer the correct three-dimensional implant coordinates from the virtual planning stage to the master model in the prosthodontic laboratory.

\section{MATERIALS AND METHODS}

Six patients were selected with different implant-prosthetic rehabilitative requirements. Three patients had a single unilateral intercalated edentulous dentition and to preserve the soft peri-implant tissue, one of these three rehabilitations was performed with a flapless 
approach. One patient exhibited a non-intercalated unilateral distal edentulous dentition, and was rehabilitated with two implant fixtures using a flapless approach and a temporary immediate load. Two patients exhibited a total maxillary edentulous dentition, and were rehabilitated with six implants via a flapless approach and temporary immediate load. In the latter case the surgical guide was used for exclusive mucosal support. Moreover, in both total maxillary edentulous dentitions, bone-residual implants were lodged in a strategically inclined way to reduce the distal cantilever and consequently to reduce the stress to which these systems are subjected [15]. The distribution of the implant sites is shown in Table 1.

Table 1 - Patients and distribution of the implant sites

\begin{tabular}{|c|c|c|c|c|c|}
\hline Patient 1 & Patient 2 & Patient 3 & Patient 4 & Patient 5 & Patient 6 \\
\hline \multirow{2}{*}{36} & \multirow{2}{*}{46} & \multirow{2}{*}{16} & $45-46$ & $\begin{array}{c}16-14-12-22- \\
24-26\end{array}$ & $\begin{array}{c}16-14-12-22- \\
24-26\end{array}$ \\
\hline
\end{tabular}

Inclusion criteria for this study were:

- The absence of any relevant systemic diseases that would contraindicate osseointegrated implant rehabilitation.

- The presence of sufficient bone volume for the placement of implant fixtures without using regenerative techniques.

- In the case of smokers, no more than 10 cigarettes for day.

\subsection{RADIOGRAPHIC STENTS}

Virtual surgical planning was achieved using softwareIMPLANT 3D by Medialab. By this means, it was possible to examine in minute detail the bony structures and the mucous profiles of the patient to evaluate the positioning of implant fixtures in a prosthetically guided manner. In respect of hard and soft tissues, it was possible to evaluate the remaining portion of hard and soft tissues after virtual positioning the implant before surgery. This planning was possible through the interpretation of DICOM images of the dental examination-scan carried out on the patient with radiographic stents suitably positioned and supported in the oral cavity. The radiography stents were produced from self-curing resin mixed with barium sulphate powder in a ratio of 3:1.

To avoid unwanted cuspal overlaps of opposing teeth and to effectively stabilize the radiographic stents during the $\mathrm{CT}$ examination, some stabilizers were made using extra-hard wax of $10 \mathrm{~mm}$ height, which patients bit occlusally between their dental arches during CT examination, (Fig. 1).

The radiographic stents constituted an important landmark to correct the diagnosis in the virtual analysis. They were developed by our dental technician using suitable diagnostic wax, in turn developed from previous aesthetic analysis. In this way it was possible to achieve 3D virtual simulations, with implant planning, not only in a prosthetically guided way, but especially in a manner that was biomechanically correct (Fig. 2). 


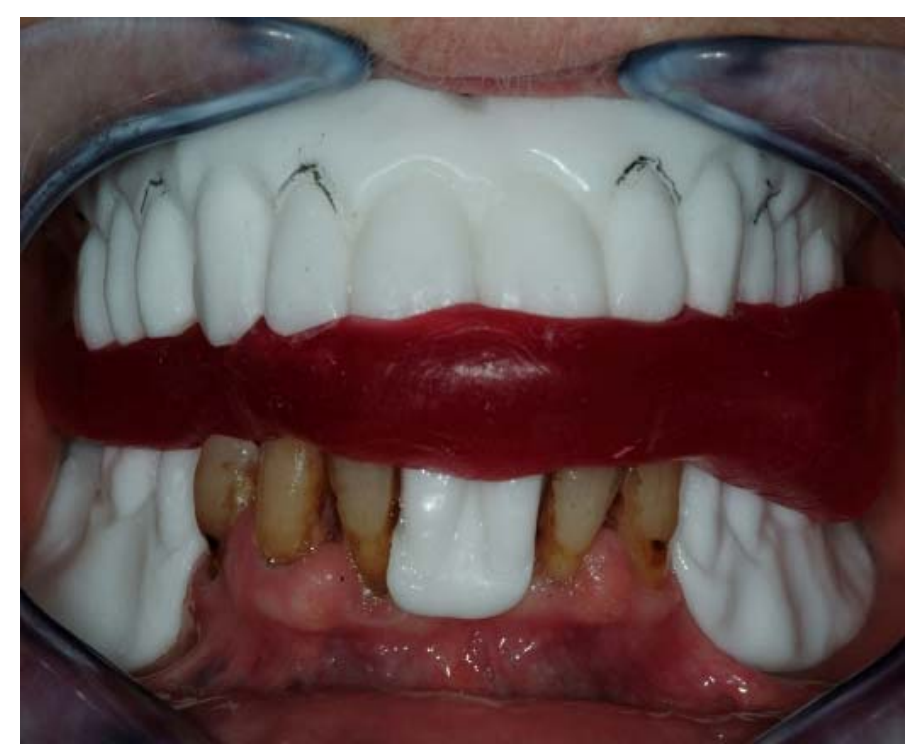

Figure 1 - Occlusal check in extra-hard wax during CT examination

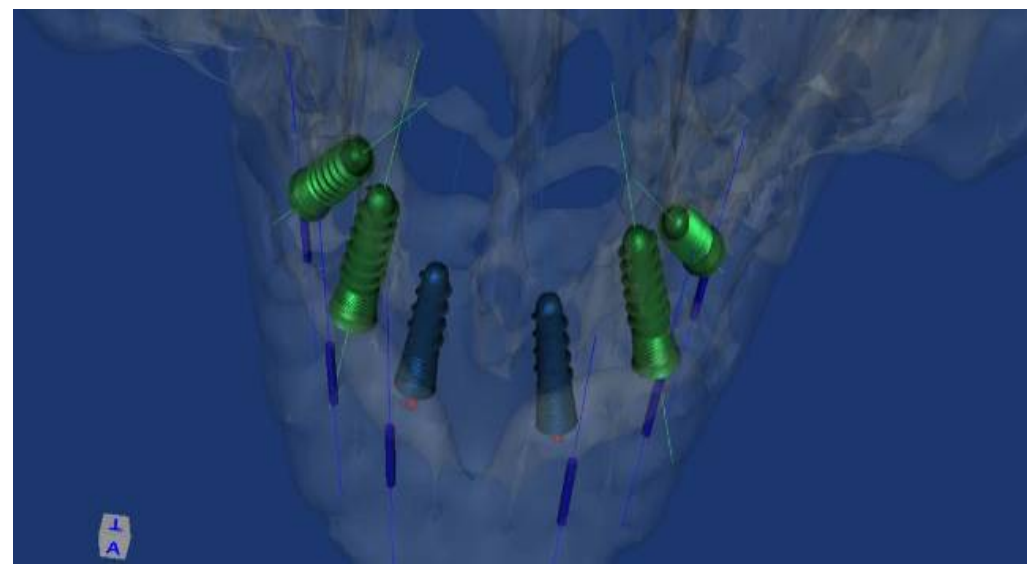

Figure 2 - Implant planning by 3D virtual simulations

\subsection{IMPRESSION TECHNIQUES}

Special care was taken to select reliable impressions and an extremely precise protocol for the support of future surgical stents. In the case of surgical stents for dental support, the impression had a mucostatic character and was performed with a one-step, two-phase technique.

The materials used for mucostatic impressions were PVS addition silicones (Poly Vinyl Siloxanes) used in the following manner. The first phase of the material (Express II, 3M) was placed exclusively in the tray while the second phase (Extrude regular, KERR) was injected only within the patient's oral cavity (Fig. 3). 


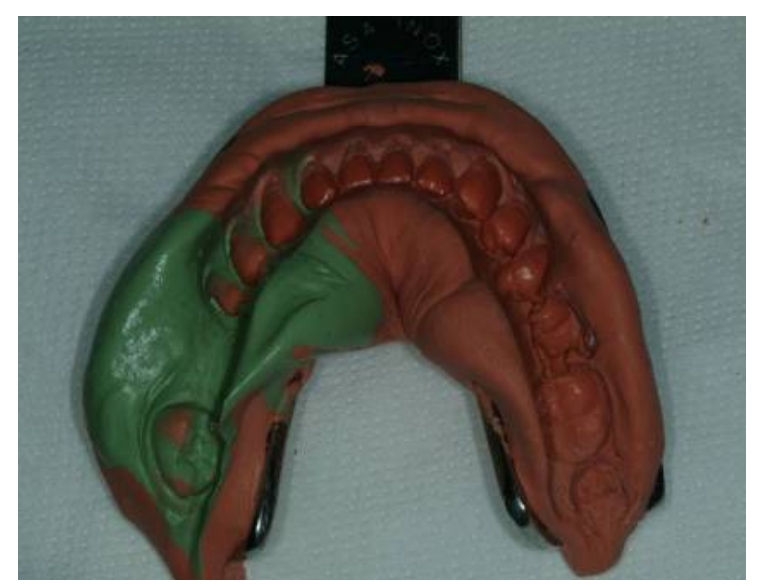

Figure 3 - Mucostatic Impression for surgical stents with dental support

In the case of surgical stents for exclusive mucosal support, the impressions were taken with an overextended muco-compressive character (Fig. 4). Alternatively, to suppress mucosal resilience in total edentulous cases, and therefore to increase the stability and the fidelity of future surgical guides with exclusive mucosal support, a biphasic impression was created in two stages.

Again, in such cases, the materials used were PVS A-silicones. For the first phase, a silicone putty-dough of heavy consistency (Extrude heavy body, KERR) was used. For the second phase a medium consistency silicone (Extrude regular, KERR) was used. This was not injected directly into the oral cavity, but placed exclusively in the tray above the heavy stage, without having previously altered the impression, as normally happens in the putty-wash technique.

This type of procedure was adopted to avoid the necessity of having to use an intrabone anchor-pin during the surgical procedure, for optimum stability of the surgical stents, when using only the mucosal support. The reason for reducing the resilience of the mucosal part was so that it would not generate a considerable margin of error.

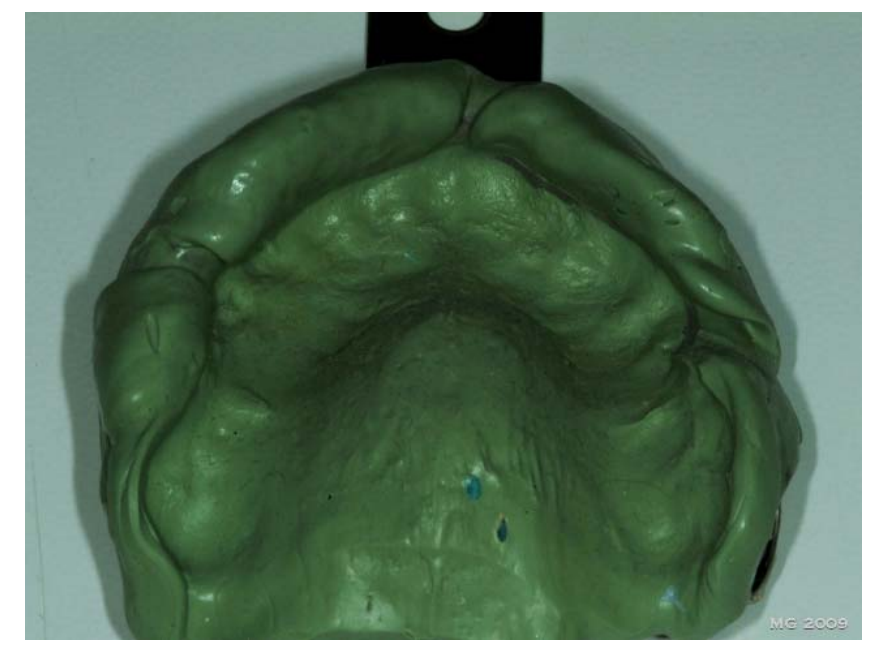

Figure 4 - Muco-compressive impressions for surgical stents with exclusive mucosal support 


\subsection{SURGICAL STENTS}

Surgical stents were fabricated using a Ray Set machine (Biaggini Medical Devices), (Fig. 5). By this it was possible to interpret the 3D data obtained from the virtual planning stage. From the virtual data, the implant coordinates were transferred to the Ray Set machine. The machine orientated the resin or plaster master model and then placed, in a correct threedimensional manner, the implant analogs in accordance with the parameters from the virtual analysis.

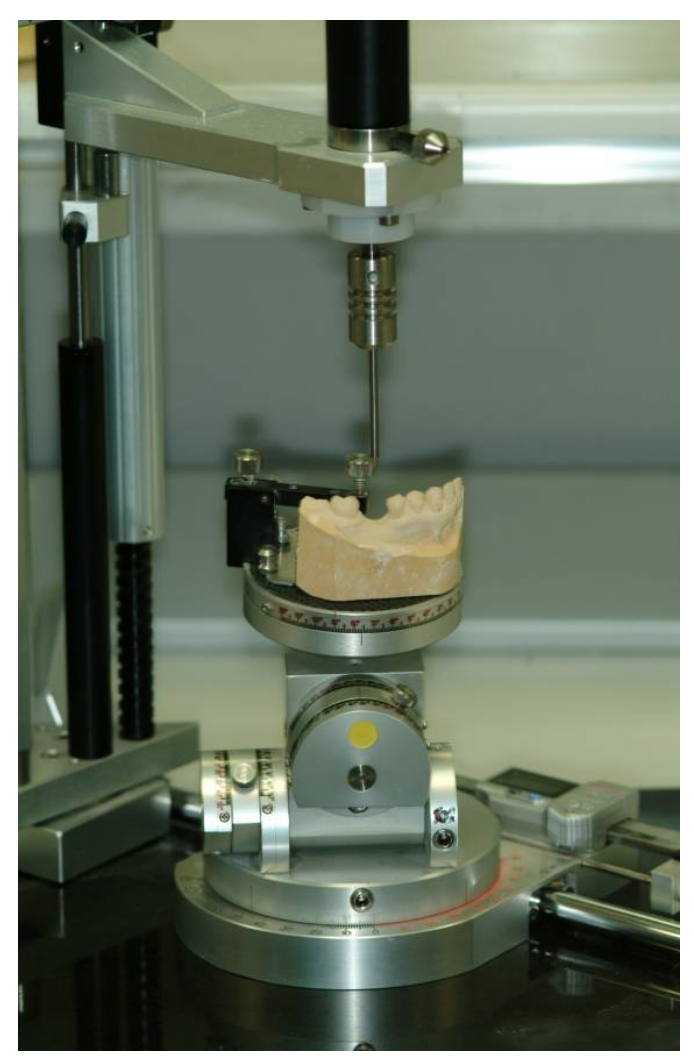

Figure 5 - Ray set machine

Once correctly positioned in the master model, the analogue implants were superimposed on the mirror-like image from virtual preoperative planning. The dental technician could then make both surgical stents with a resinous framework like total removal dentures (see stents for exclusive mucosal support), and surgical stents with a metal core like a skeleton (see stents at the exclusive dental support).

An important aspect was the possibility of making customizations at the surgeon's request where, for periodontal reasons, it may be convenient to support and protect the access flaps (Fig. 6). 


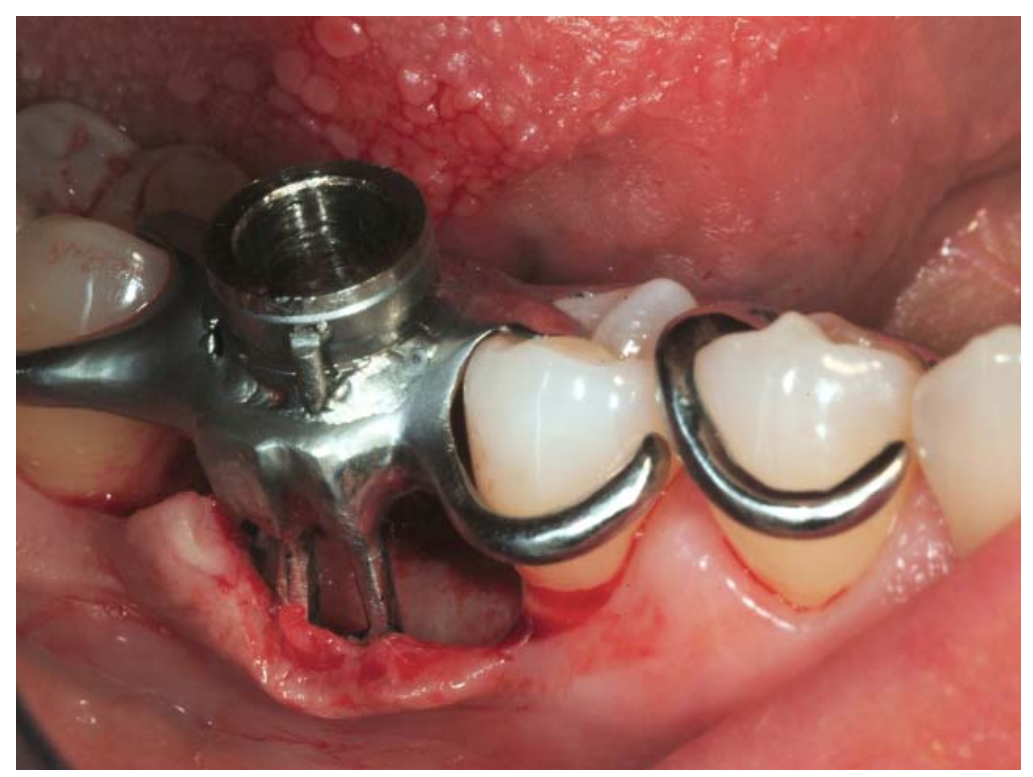

Figure 6 - Customizations to support and protect the access flaps

\subsection{SURGICAL AND PROSTHETIC PROTOCOL}

Seventeen conical implants were used with lengths between 11 and $13 \mathrm{~mm}$ (EXACTA WP, Biaggini Medical Devices), to obtain the best primary stability possible, which however was always above $45 \mathrm{~N}$.

For the same reason, the implant sites on the upper jaw were all under-prepared. On the basis of periodontal biotype a flapless approach was chosen. In the case of a thick biotype, and an approach with flap in the case of thin biotype, in order to preserve the keratinized gingiva.

The number of implants, the length and type of surgical approaches, are summarized in the Table 2.

Table 2 - Number and length of implants and type of surgical approach

\begin{tabular}{|l|c|c|}
\hline & Flapless & With Flap \\
\hline Implant length $11 \mathrm{~mm}$ & 4 & 1 \\
\hline Implant length $13 \mathrm{~mm}$ & 11 & 1 \\
\hline
\end{tabular}

All implants received the provisional immediate load within 48 hours post-surgery. In the case of the individual intercalated edentulous patients the prosthesis was attached through the abutment millable prosthesis screwed with a torque of $30 \mathrm{~N} \bullet \mathrm{cm}$, and using a cemented resin crown. The undisputed advantage of being able to use a presurgical master model was the possibility for the clinician to have a provisional prosthetic device immediately after surgery, substantially shortening the time before prosthetic loading. 
In the case of total edentulism a provisional immediate load with screwed metal mesostructure and resin superstructure was used, after having taken a suitable master impression immediately after surgery (Fig. 7).

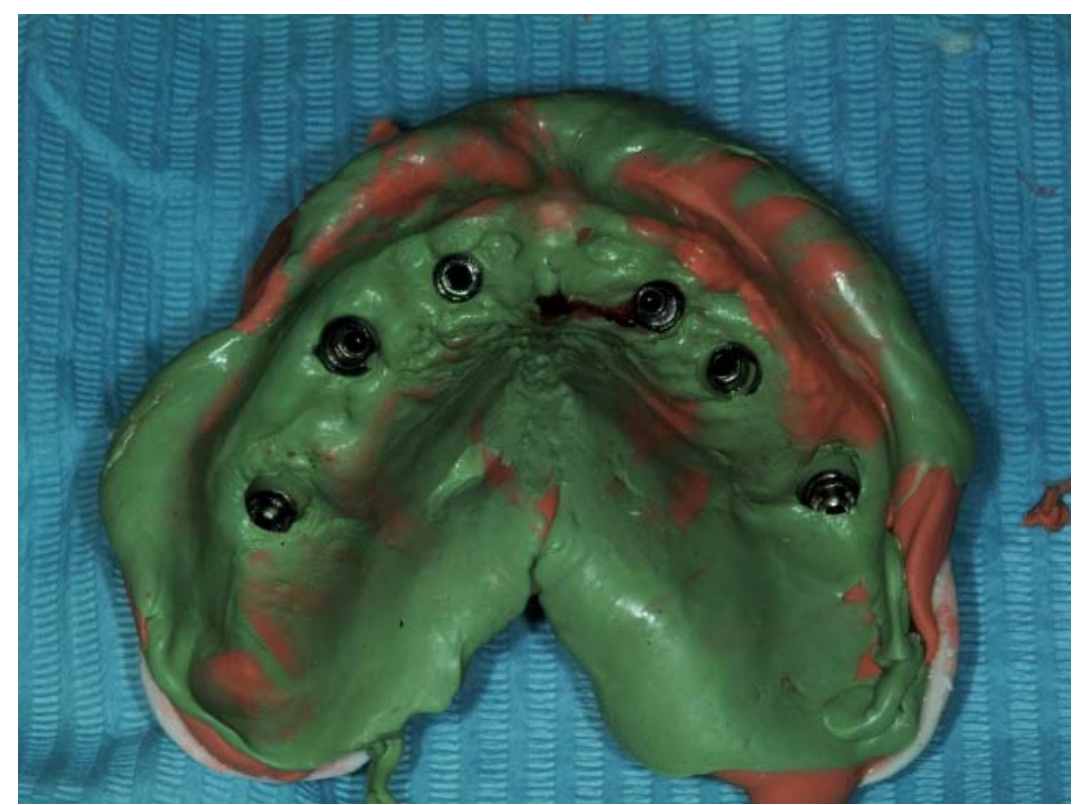

Figure 7 - Master impression immediately after surgery in the case of total edentulism

The taking of the master post-surgical impressions was greatly facilitated by the prosthetic planning performed on the master pre-surgical model. By this means it was possible to prepare the splinting resin, the individual tray (Fig. 8) and even the prosthetic pre-angular components (EXE WP, Biaggini Medical Devices) able to compensate and correct for the implant dis-parallelism (Fig. 9).

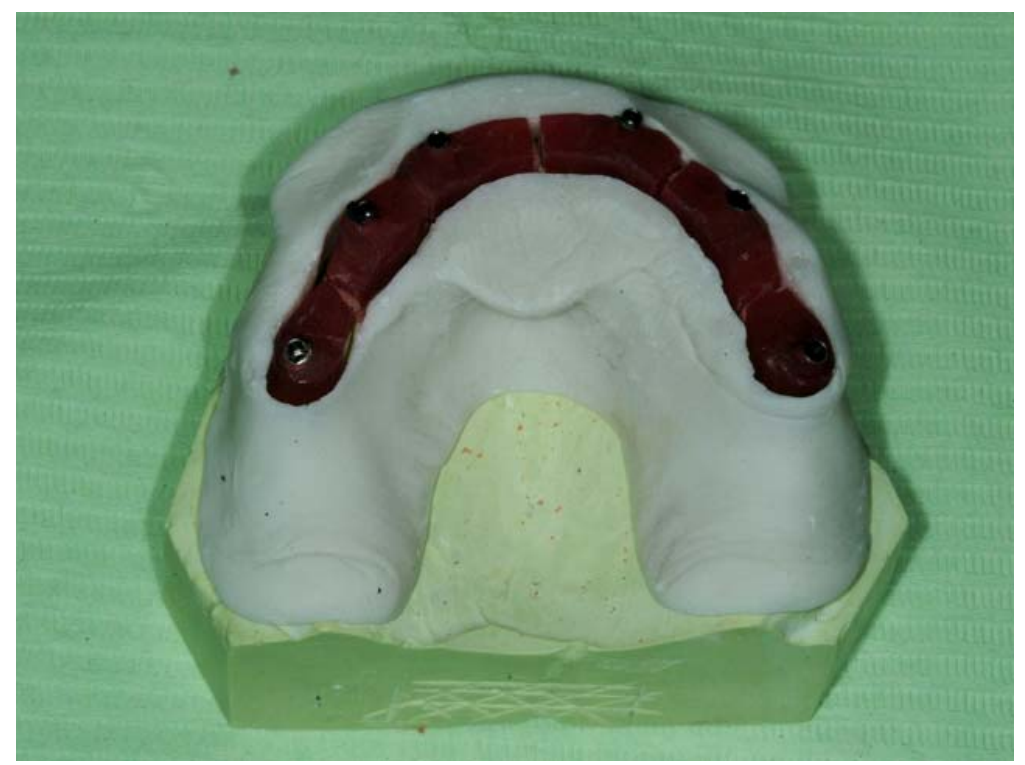

Figure 8 - Prosthetic planning performed on the master presurgical model: splinting resin, individual spoon 

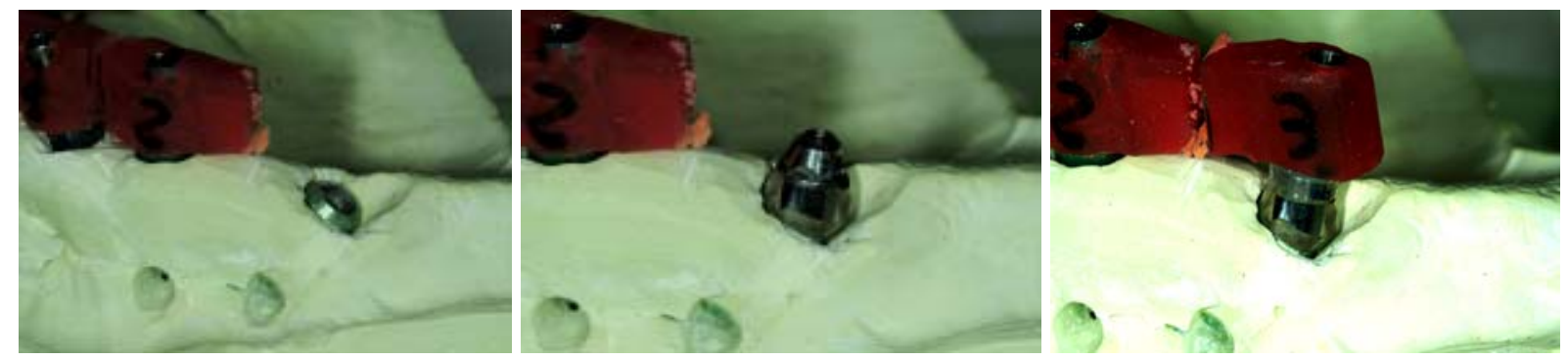

Figure 9 - Prosthetic preangular components on the master presurgical model able to compensate and correct for the implant dis-parallelism.

\subsection{POST-OPERATIVE RADIOGRAPHS AND FOLLOW UP}

Immediately after surgery and two years after surgery, control intraoral radiographs were made, to superimpose and compare, in the virtual environment, linear measurements obtained from the digital postoperative radiographs.

These radiographs were acquired on-line by computer with the digital sensor (RVG, Trophy). Thereby it was possible to obtain two-dimensional feedback with a margin of error of 9\% [16]. However, for verification of this percentage, it was essential to adopt the parallel technique, according to which the digital sensor and the dental element, namely the implant to be radiographed must be positioned perpendicularly to the axis of the X-ray tube.

Concurrently with the digital radiographic examinations, starting from three months after surgery, clinical examinations were performed designed to highlight any peri-implant problems, such as lack of osseointegration in the first place, and secondly, any peri-implantitis with loss of peri-implant bone support.

\section{RESULTS}

Comparing linear measurements performed with digital radiographs, through Trophy Windows software, and those performed in the virtual preoperative planning by Implant 3D software, a margin of error of about $0.2 \mathrm{~mm}$ was recorded (Figures 10-14).

The linear measurements were used to calculate the distance between each implant apex and the relevant anatomical structures, such as the mesial margin of the mandibular canal and the maxillary sinus.

After three months intraoral clinical and radiographic examinations, the successful osseointegration of all implants was confirmed. This osseointegration has persisted over the two following years, a period during which each patient has been monitored every six months. 


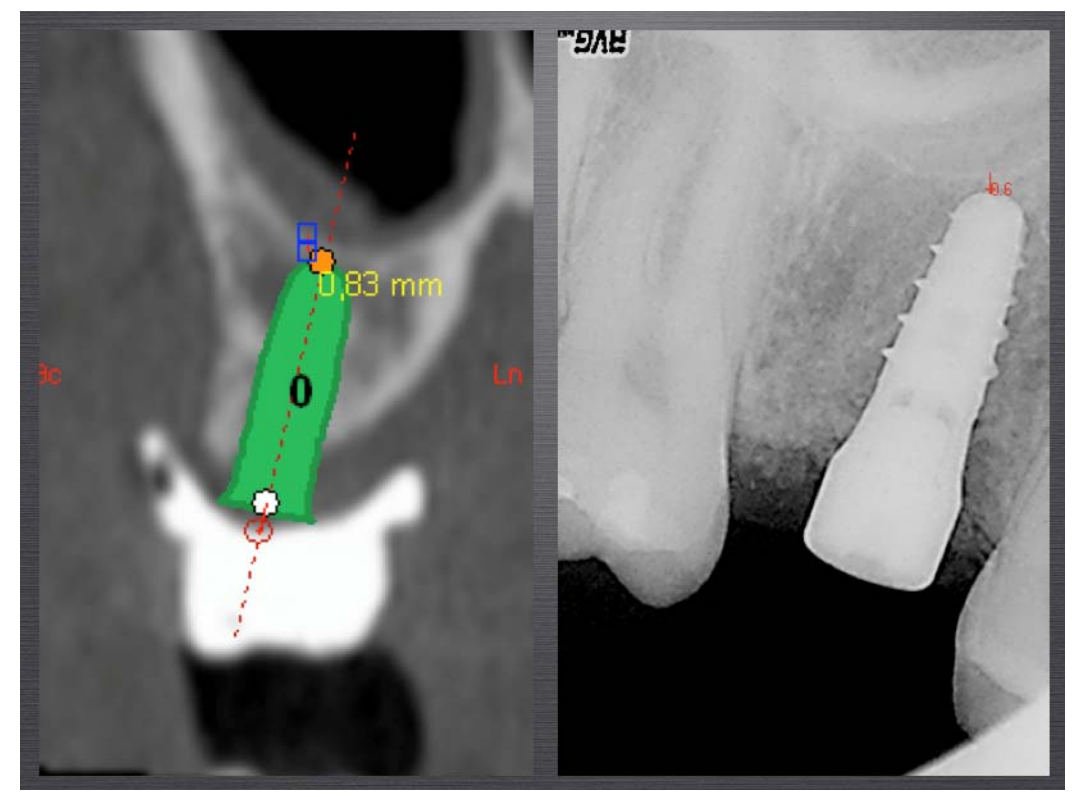

Figure 10 - Comparison between linear measurements performed with digital radiographs and those performed during virtual preoperative planning

The follow-up examination at two years (Figures 15-16), moreover, highlighted the absence of complications such as peri-implantitis or peri-implant loss of bone support. To this must be added, both the proper support of the interdental gingival papillae, and those that exist between the tooth and implant, and the proper support of the gingival papillae interimplant, namely those ones between adjoining implants.

The results of the comparisons are shown in Table 3.

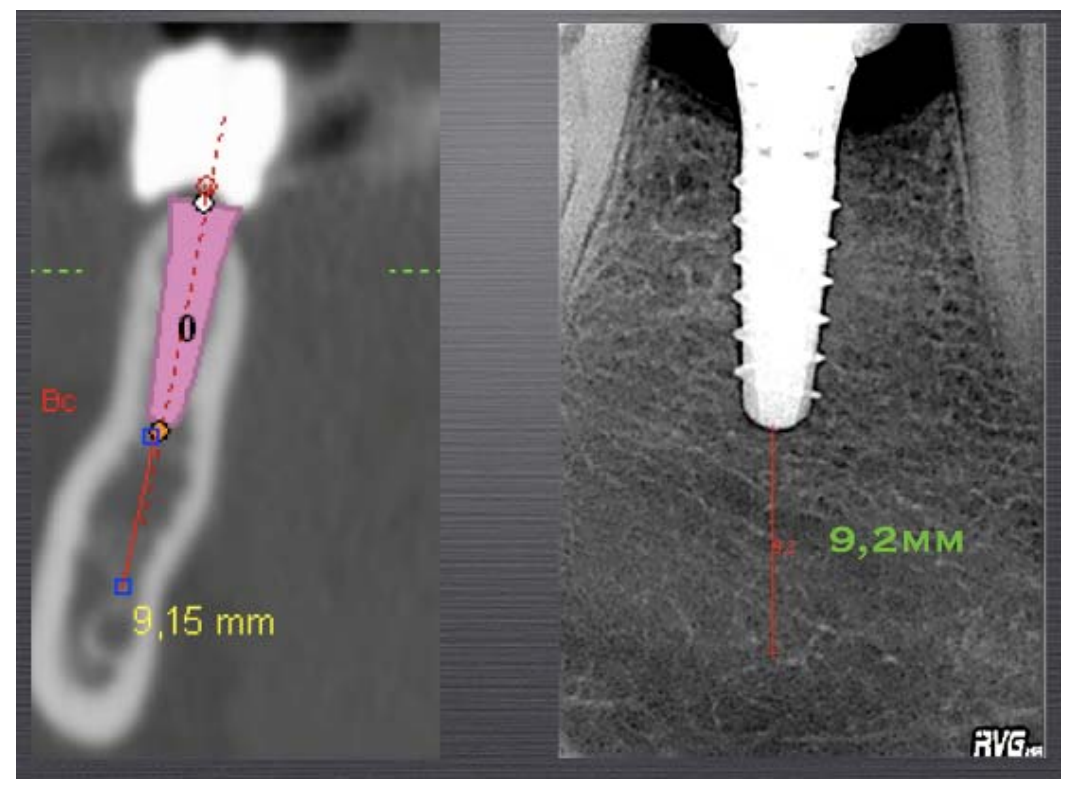

Figure 11 - Comparison between linear measurements performed with digital radiographs and those performed in the virtual preoperative planning 


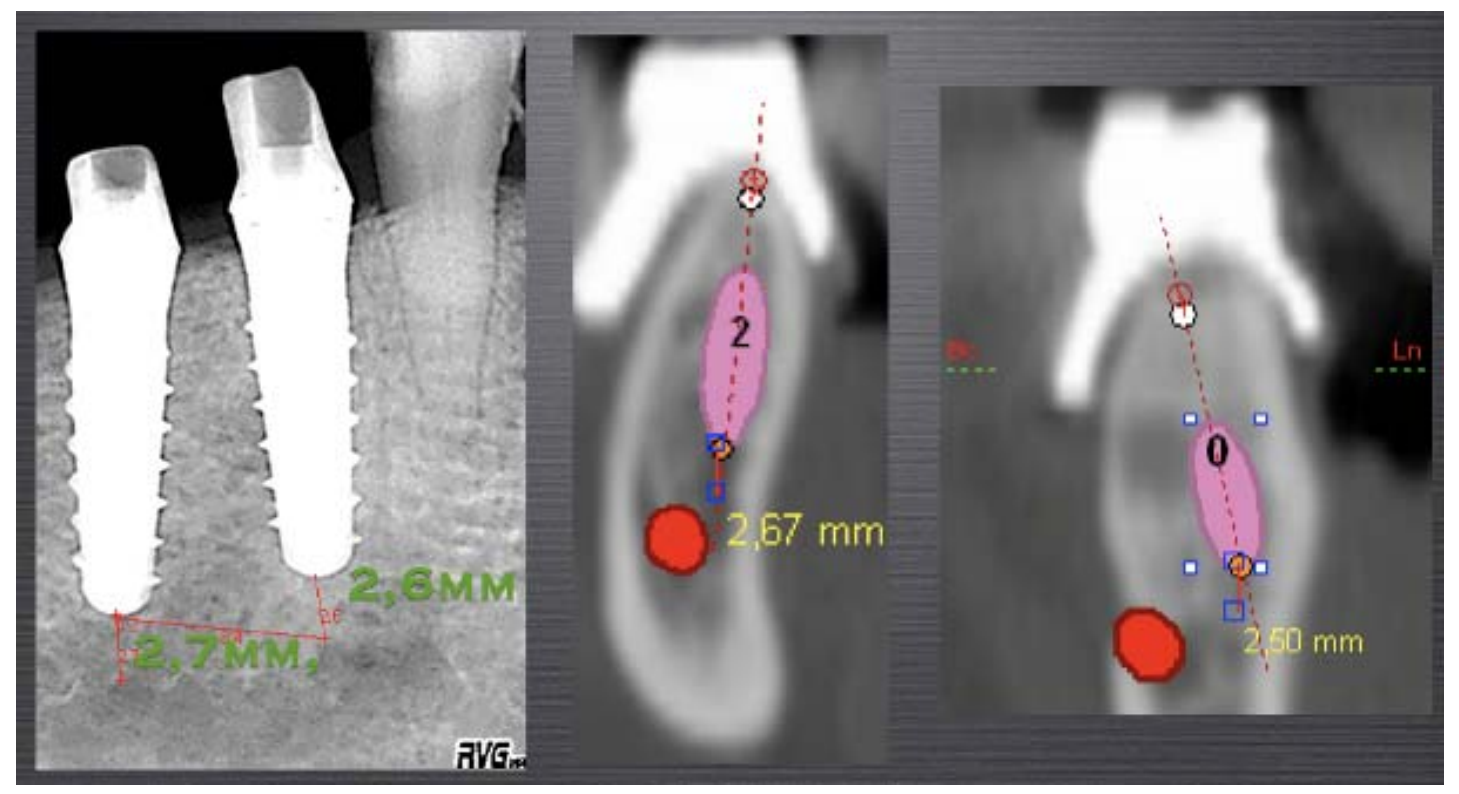

Figure 12 - Comparison between linear measurements performed with digital radiographs and those performed in the virtual preoperative planning

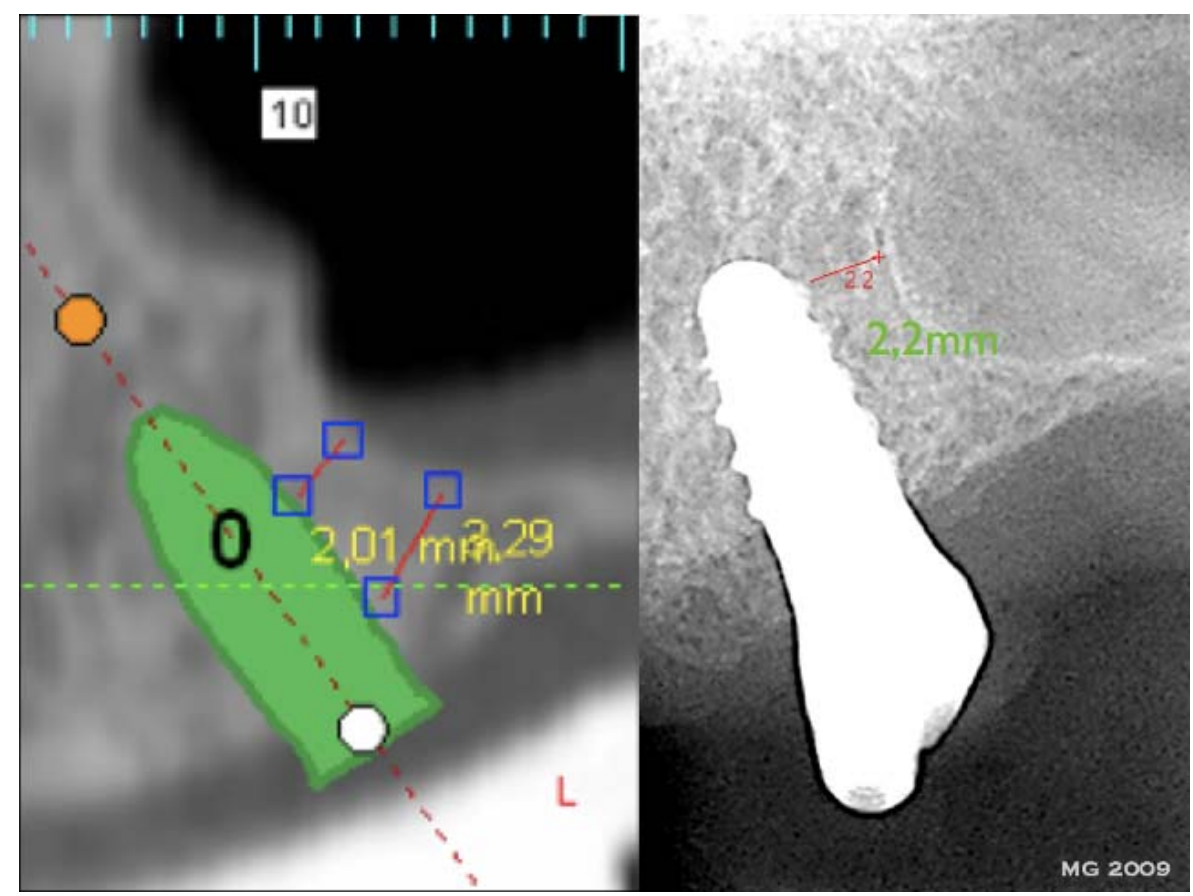

Figure 13 - Comparison between linear measurements performed with digital radiographs and those performed in the virtual preoperative planning 


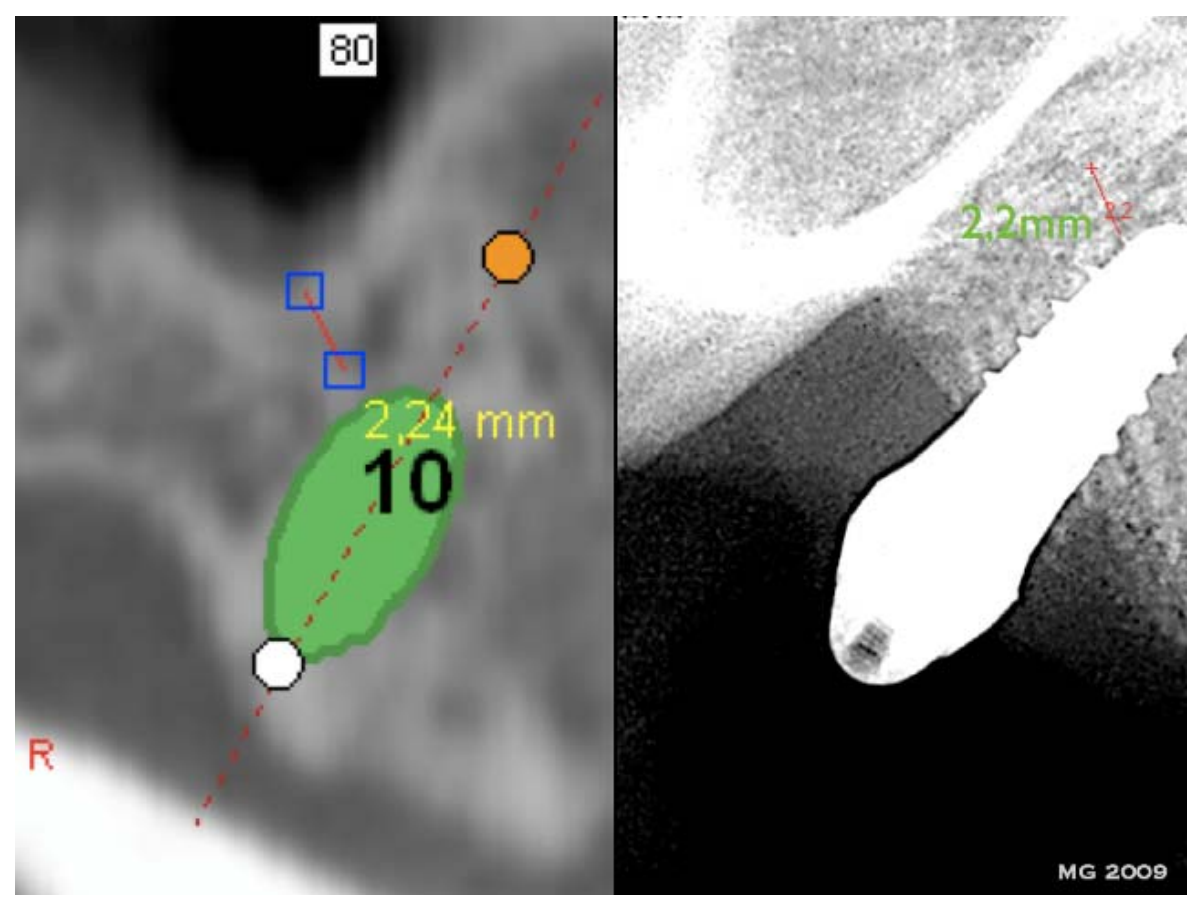

Figure 14 - Comparison between linear measurements performed with digital radiographs and those performed in the virtual preoperative planning

Table 3 - Results of the comparison between linear measurements performed in the virtual preoperative planning and those performed with digital postoperative radiographs

\begin{tabular}{|l|c|c|c|}
\cline { 2 - 4 } \multicolumn{1}{c|}{} & Virtual Pre-operative & RX Digital Post-operative & Margin of error \\
\hline Fig. 10 & $0.83 \mathrm{~mm}$ & $0.6 \mathrm{~mm}$ & $0.23 \mathrm{~mm}$ \\
\hline Fig. 11 & $9.15 \mathrm{~mm}$ & $9.2 \mathrm{~mm}$ & $0.05 \mathrm{~mm}$ \\
\hline Fig. 12 & $2.7 \mathrm{~mm}-2.6 \mathrm{~mm}$ & $2.67 \mathrm{~mm}-2.5 \mathrm{~mm}$ & $0.03 \mathrm{~mm}-0.1 \mathrm{~mm}$ \\
\hline Fig. 13 & $2.01 \mathrm{~mm}$ & $2.2 \mathrm{~mm}$ & $0.2 \mathrm{~mm}$ \\
\hline Fig. 14 & $2.24 \mathrm{~mm}$ & $2.2 \mathrm{~mm}$ & $0.04 \mathrm{~mm}$ \\
\hline
\end{tabular}




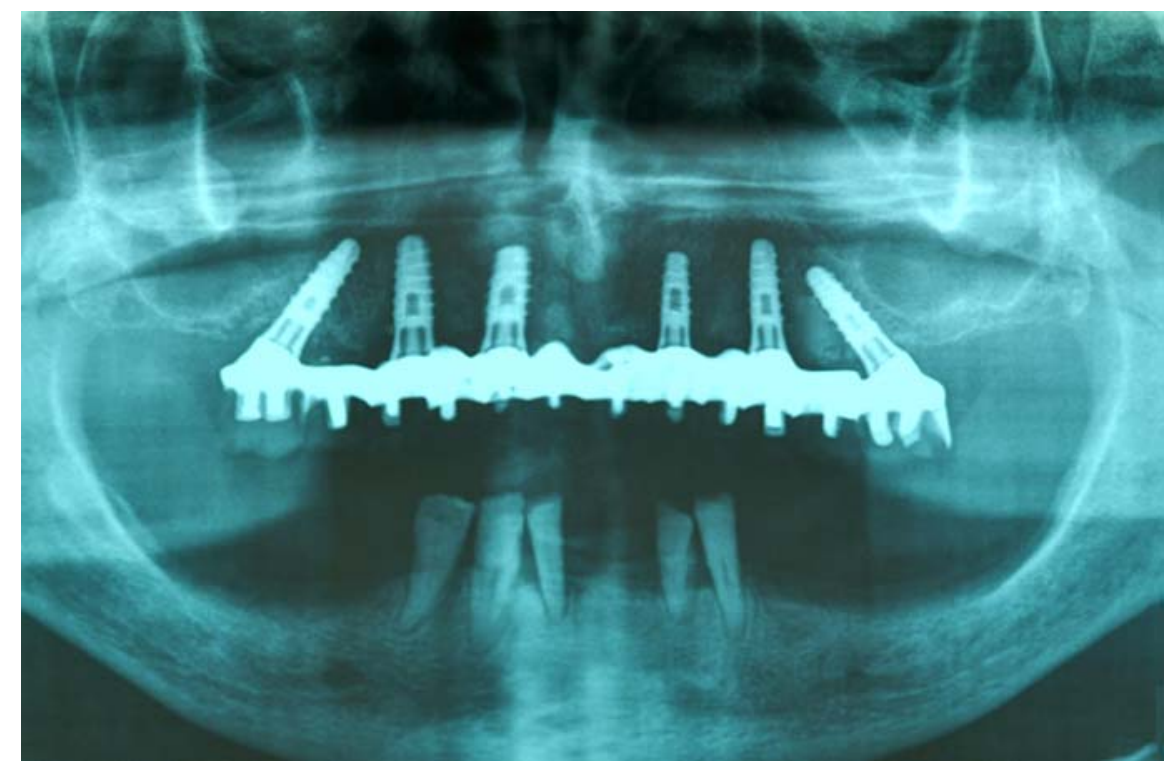

Figure 15 - Orthopantomography after two years. Full Maxillary Arch
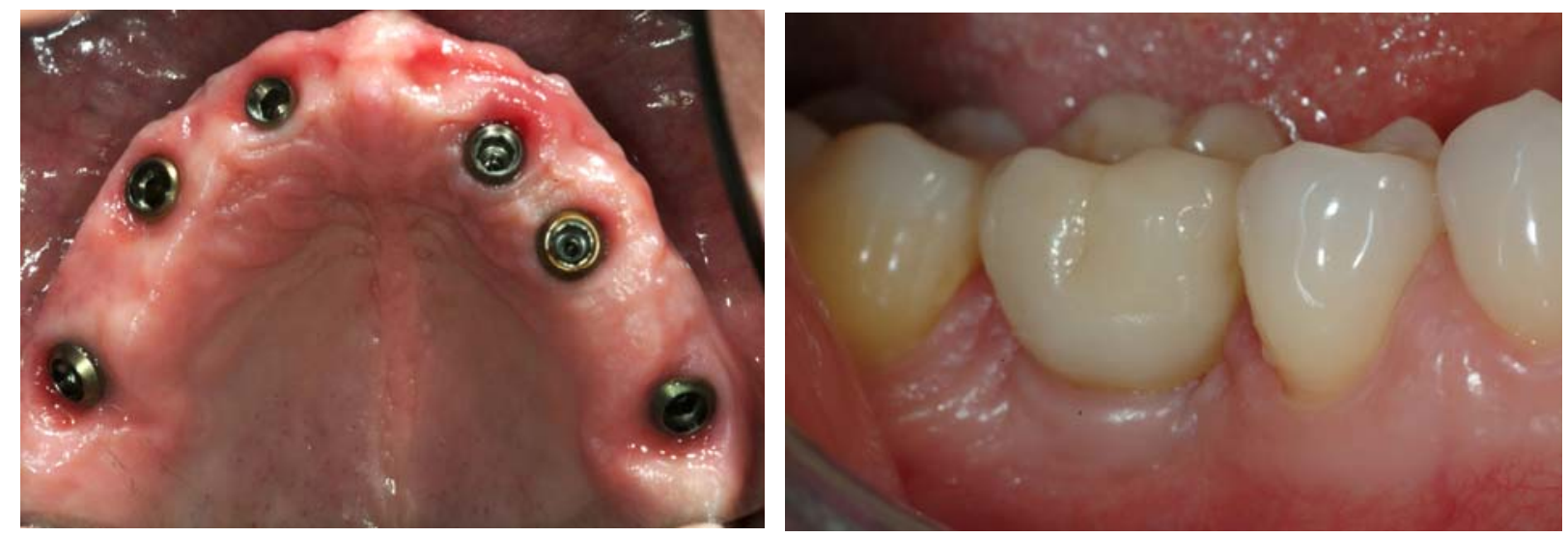

Figure 16 - Clinical images with follow-up for two years

\section{DISCUSSION}

The functional and aesthetic success of osseointegrated implant oral rehabilitation is strictly dependent on correct preoperative planning. Prior to application of Virtual Prototyping in Dentistry, such planning has been carried out exclusively with the aid of two-dimensional $\mathrm{X}$-ray images, such as osseo-pantomography and intraoral X-rays, or with large images from three-dimensional examinations, but examined exclusively on film by transillumination through a diaphanoscope.

With the aid of Virtual Prototyping in the field of implant surgery, starting from CT Dicom data, it is possible to plan very detailed surgery in a virtual environment. So it is possible pre-operatively, with three-dimensional virtual analysis, to insert implants in the most strategically valid locations, carefully considering the availability of bone from both a quantitative and qualitative point of view. Also the choices of the surgeon in a virtual environment 
can be guided by biomechanical and prosthetic assessments, as this aspect of oral osseointegrated rehabilitation appears to be fundamental.

According to various authors [17-19], the accuracy obtainable in implant positioning, by means of computer-assisted procedures, is superior to that obtained by a freehand approach. However this outcome is closely dependent on the accuracy with which the data are transferred pre-operatively from the virtual planning to the master model.

In this regard special consideration must be given to the positioning of the implant analogues on the plaster cast in the preoperative master model. With the Ray Set technique, the implant analogues are not positioned with the aid of stereolithographic surgical guides, but it is the machine itself that, after having properly oriented the model according to the spatial coordinates from the software, allows the dental technician to position implant analogs correctly oriented and with the right depth.

The depth of the implant analogs in the master model is determined by measuring the thickness of the mucosa of the patient in the virtual design phase. Therefore it is not the stent that determines the position of the implants, but it is the position of the latter which allows the construction of the surgical stent. This aspect is crucial, because the technician can perform customizations at the request of the surgeon if, for periodontal reasons, it is preferable to support and protect the access flaps.

Another very effective aspect, has been the technique of muco-compressive impressions performed on the edentulous jaw to eliminate the error arising from the mucosal resiliency itself. With this approach, it has become unnecessary to use an endosseous anchor-pin to stabilize the surgical stent (Fig. 17). In fact, in both full-arch interventions considered in this study, the surgical stent was greatly stabilized by exploiting the exclusive mucosal support.
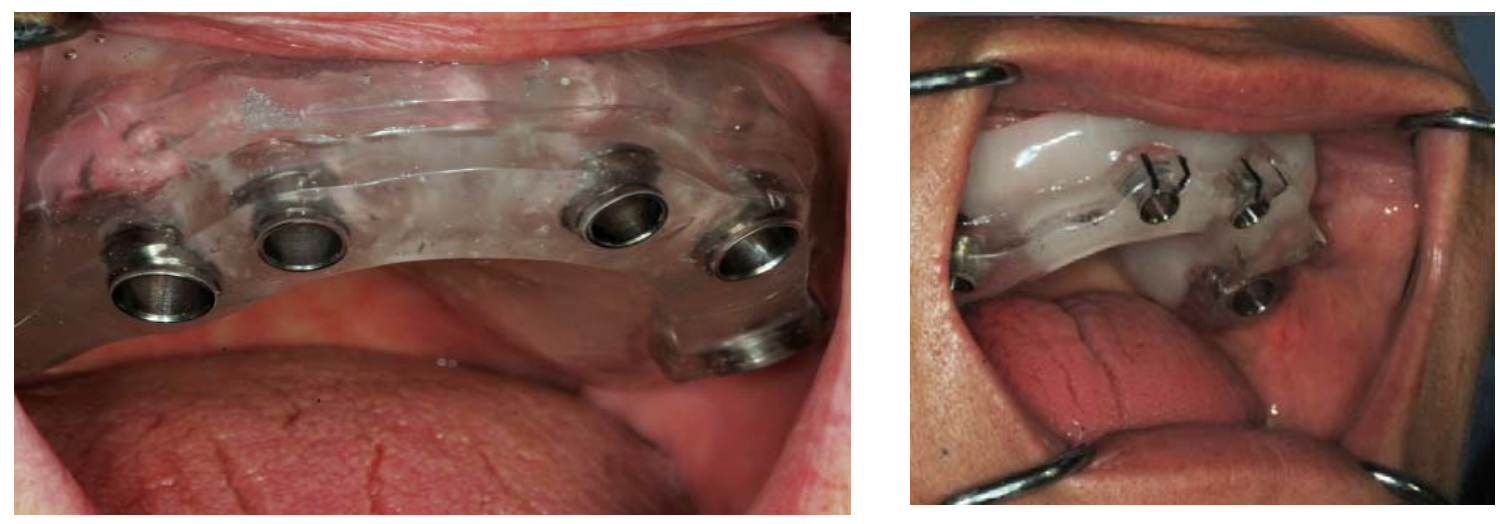

Figure 17 - Technique of muco-compressive impression performed on an edentulous jaw

This technique makes the surgery less invasive and offers the patient a smoother postoperative recovery.

Other factors can, however, affect the accuracy with which data are transferred from the virtual to the surgical environment. Widmann, Pattersson et al. $[17,18]$ consider that the thickness of the engraving, the patient's movements and the scanning parameters can generate inaccuracies during image acquisition. Furthermore, human error remains an uncontrollable factor which can contribute to some additional imprecision. 


\section{CONCLUSIONS}

Despite the limitations of the method used for feedback between the three-dimensional virtual design and the two-dimensional postoperative digital X-rays, we conclude that our computer-designed surgical guides represent a valuable aid for the implant surgeon. By virtual planning, using the instrument for transfer of implant coordinates and by techniques of muco-static and muco-compressive impressions, a minimally invasive implant surgery can be performed that is biomechanically and prosthetically guided.

Further studies, using, for example, Reverse Engineering systems, will be useful to verify the accuracy with which the position of implant analogs for plaster of the pre-surgical master model reflect the position of implant analogs present in the post-surgery master model.

\section{ACKNOWLEDGEMENT}

The authors wish to thank Jonata laboratory for the manufacture of radiological and surgical stents, Capezzuto laboratory for pre-operative aesthetic analysis and Stravino laboratory for the intercalated rehabilitations.

Competing interests: None declared

Funding: None

Ethical approval: Not required

\section{REFERENCES}

[1] Aglietta M, Siciliano VI, Zwahlen M, et al. A. systematic review of the survival and complication rates of implant supported fixed dental prostheses with cantilever extensions after an observation period of at least 5 years. Clin Oral Implants Res 2009; 20:441-451.

[2] Jung RE, Pjetursson BE, Glauser R, Zembic A, Zwahlen M, Lang NP. A systematic review of the 5-year survival and complication rates of implant-supported single crowns. Clin Oral Implants Res 2008; 19:119-1.30.

[3] Pjetursson BE, Tan K, Lang NP, Bragger U, Egger M, Zwahlen M. A systematic review of the survival and complication rates of fixed partial dentures (FPDs) after an observation period of at least 5 years. Clin Oral Implants Res 2004; 15:667-676.

[4] Bianco J,Carral C, Linares A,Perez J,Munoz F. Soft tissue dimension in flapless immediate implants with and whithout immediate loading: an experimental study in beagle dog. Clin Oral Implants Res 2011

[5] Bianco J,Linares A,Villaverde G,Perez j,Munoz F. Flapless immediate implant placement with or without immediate loading: a histomorphometric study in beogle dog. $\mathrm{J}$ Clin Periodontol 2010

[6] Rousseau P. Flapless and traditional dental implant surgery: an open, retrospective comparative study. J Oral Maxillofac Surg 2010

[7] Garg AK, Vicari A. Radiographic modalities for diagnosis and treatment planning in implant dentistry. Implant Soc 1995; 5:7-11. 
[8] Lam EW, Ruprecht A, Yang J. Comparison of twodimensionai orthoradially reformatted computed tomography and panoramic radiography for dental implant treatment planning. J Prosthet Dent 1995; 74:42-46.

[9] Sakakura CE, Morais JA, Loffredo LC, Scaf G. A survey of radiographic prescription in dental implant assessment. Dentomaxillofac Radiol 2003; 32:397-400.

[10] White SC, Heslop EW, Hollender LG, Mosier KM, Ruprecht A, Shrout MK. Parameters of radiologìc care: an official report of the american academy of oral and maxillofacial radiology. Oral Surg Oral Med Oral Pathol Oral Radiol Endod 2001; 91:498-511.

[11] Aranyarachkul P, Caruso J, Gantes B, et al. Bone density assessments of dental implant sites: 2. Quantitative conebeam computerized tomography. Int j Oral Maxillofac Implants 2005; 20:416-424.

[12] Bolin A, Eliasson S, von Beetzen M, Jansson L. Radiographic evaluation of mandibular posterior implant sites: correlation between panoramic and tomographic determinations. Clin Oral Implants Res 1996; 7:354-359.

[13] Gray CF, Redpath TW, Bainton R, Smith FW. Magnetic resonance imagíng assessment of a sinus lift operation using reoxidised cellulose (Surgicel) as graft material. Clin Oral Implants Res 2001; 12:526-530.

[14] Gahleitner A, Podesser B, Schick S, Watzek G, Imhof H. Dental CT and orthodontic implants: ímaging technique and assessment of available bone volume in the hard palate. Eur J Radiol 2004; 7:257-262.

[15] Bevilaqua M,Tealdo T,Menini M,Pera F,Mossolov A,Drago C,Pera P. The influence of cantiliver length and implant inclination on stress distribution in maxyllary implantsuppoprted fixed dentures.J Prosthet Dent. 2011;105:5-13

[16] Lazzerini F, Minorati D, Nessi R, Gagliani M, Uslenghi CM. The measurement parameters in dental radiography: a comparison between traditional and digital technics.Radiol Med. 1996 Apr;91(4):364-9. Italian.

[17] Widmann G, Baie RJ. Accuracy in computer-aided implant surgery - a review. Int J Oral Maxillofac Implants 2006; 21:305-313.

[18] Pettersson A, Komiyama A, Hultin M, Nasstróm K, Klinge B. Accuracy of virtually planned and template guided implant surgery on edentate patients. Clin Implant Dent Relat Res 2010; May 11. DOI: 10.1111/j.1708-8208.2010.00285.x. [Epub ahead of print].

[19] Stumpel LJ. Deformation of stereolithographicaliy produced surgical guides: an observational case series report. Clin Implant Dent Relat Res 2010; Feb li. DO!: 10.1111/ j.1708-8208.2010.00268.x. [Epub ahead of print]. 